\title{
Advanced Ultra-sharp Fano filter resonances in integrated photonic resonators with coupled Sagnac loop reflectors
}

\author{
Hamed Arianfard, Jiayang Wu, and David J. Moss* \\ Optical Sciences Centre, Swinburne University of Technology, Hawthorn, VIC 3122, Australia.
}

\section{Introduction}

Abstract: We investigate Fano resonances in integrated photonic resonators formed by coupled Sagnac loop reflectors (CSLR). Mode splitting in the CSLR resonator is tailored to achieve multiple Fano-like resonances with ultrahigh extinction ratios and slope rates.

Fano resonances feature an asymmetric profile in resonant lineshape that are a fundamental phenomenon induced by interference between a discrete localized state and a continuum state [1-3]. It was first reported early in the $20^{\text {th }}$ century $[4,5]$, and has been widely used in atom spectroscopy since then [2]. Recent progress in photonics and nanotechnology has led to many optical phenomena analogous to Fano resonances, which have found wide applications in optical switching, sensing, light focusing beyond the diffraction limit, data storage, topological optics, and many others [1, 3, 6-8]. To date, these optical phenomena have been demonstrated in various types of resonant cavities such as dielectric rods, disordered structures, integrated photonic resonators, lattices of nanospheres, and metasurfaces [1-3, 7-9]. Amongst them, integrated photonic resonators fabricated via complementary metal-oxide-semiconductor (CMOS) technology provide competitive advantages of compact device footprint, high stability, high scalability, and low-cost mass production.

Recently $[10,11]$ we fabricated devices based on this approach in silicon-on-insulator (SOI) nanowires formed by coupled Sagnac loop reflectors (CSLR). Here, we theoretically investigate more advanced filter structures using similar principles. By adjusting the reflectivity of the Sagnac loop reflectors (SLRs) as well as the coupling strength between the SLRs and bus waveguides, we tailor mode splitting in the CSLR resonators to achieve multiple Fano resonances with ultrahigh slope rates (SRs) and extinction ratios (ERs). We theoretically show that a high ER of $\sim 64 \mathrm{~dB}$ and a corresponding slope rate of $\sim 751 \mathrm{~dB} / \mathrm{nm}$ can be achieved for a CSLR resonator with three coupled SLRs. These results highlight the strong potential of using CSLR resonators to achieve high-performance Fano-like resonances.

\section{Device configuration}

The CSLR resonators are illustrated in Figs. 1(a) and (b). The first device consists of two parallel SLRs coupled to a top bus waveguide and is termed a parallel CSLR resonator, while the second device consists of three inversely coupled SLRs and is termed a zig-zag CSLR resonator. To simplify the comparison, we assume that the SLRs are identical, and that each SLR is formed by a self-coupled SOI nanowire waveguide with a length of

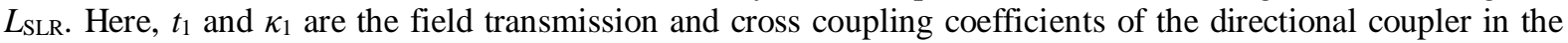
SLRs, respectively, while $t_{2}$ and $\kappa_{2}$ are the field transmission and cross coupling coefficients of the directional coupler formed by the SLRs and the bus waveguides. The lengths of the bus waveguides between the SLRs are denoted by $L_{i}(i=1,2,3,4)$. For the SOI nanowires with a cross section of $500 \mathrm{~nm} \times 260 \mathrm{~nm}$ (Fig. 1(c)). We use a waveguide group index for TE polarization of $n_{\mathrm{g}}=4.3350$ and a propagation loss of $\alpha=55 \mathrm{~m}^{-1}$, or 2.4 $\mathrm{dB} / \mathrm{cm}$, based on our fabricated devices $[10,11]$. The scanning electron microscope (SEM) image of a fabricated SLR is shown in Fig. 1(d). In the CSLR resonators, the bus waveguides introduce additional feedback paths for coherent optical mode interference, which yield the Fano resonances. The transmission spectra of the CSLR resonators can be tailored by changing the reflectivity of the SLRs and the coupling strength between the SLRs and bus waveguides. This enables flexible spectral engineering with the CSLR resonators for many applications.

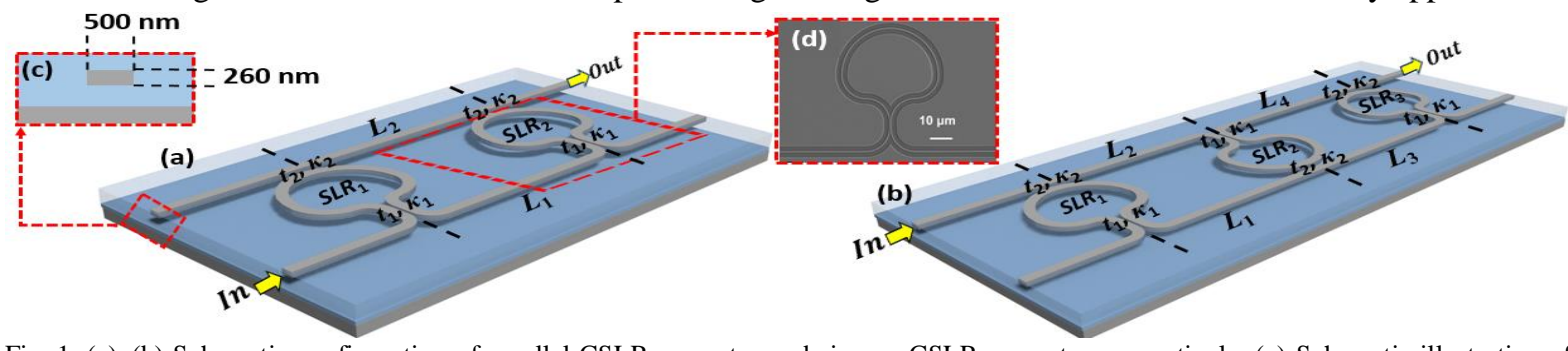

Fig. 1. (a)-(b) Schematic configuration of parallel CSLR resonator and zig-zag CSLR resonator, respectively. (c) Schematic illustration of the cross-section of the SOI nanowire waveguide. (d) SEM image of the fabricated SLR on an SOI wafer.

\section{Results and discussion}

We use the scattering matrix method $[10,11]$ to calculate the transmission spectra of the proposed CSLR resonators. Figure 2(a) shows the calculated power transmission spectrum of the parallel CSLR resonator when $L_{S L R}=L_{1,2}=113.75 \mu \mathrm{m}, t_{1}=0.74$, and $t_{2}=0.94$. As can be seen, there are multiple Fano resonances with asymmetric resonant lineshapes. As shown in Figs. 2(b) and (c), the transmission spectrum can be tailored by changing $t_{1}$ and $t_{2}$, which allows us to optimize the filter shape. Figures 2(d) and (e) show the calculated SR and insertion loss (IL) as functions of $t_{1}$ and $t_{2}$, reflecting the trade-off between SR and IL. 

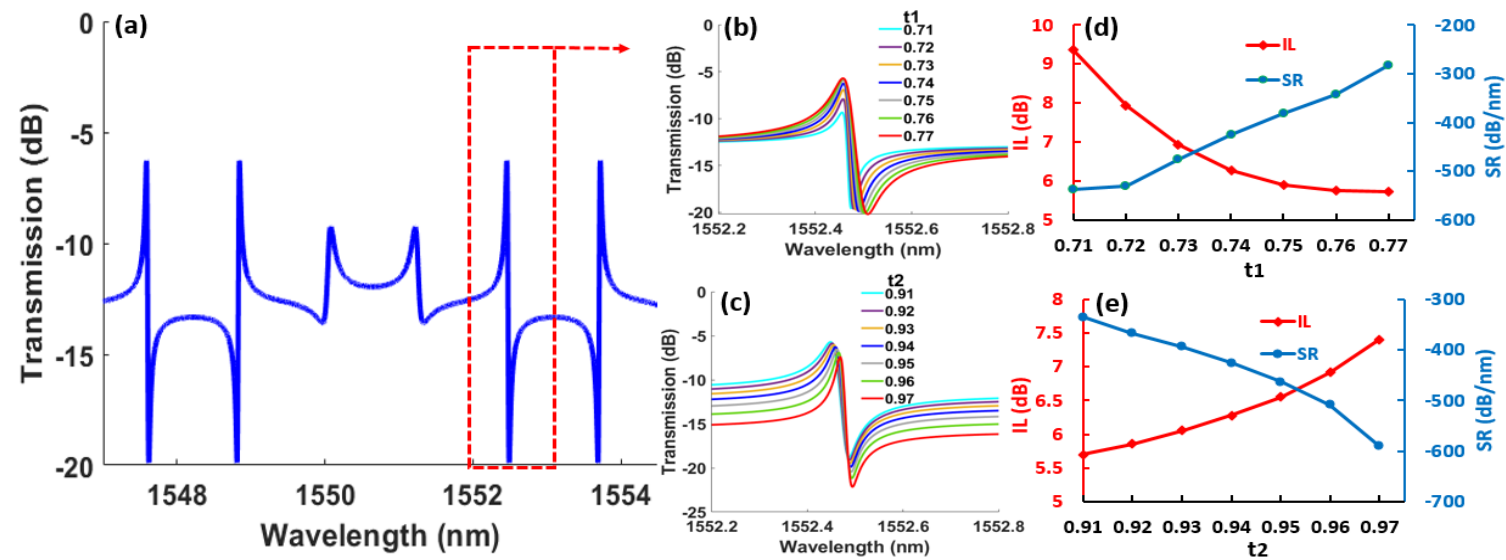

Fig. 2. (a) Calculated power transmission spectrum of the parallel CSLR resonator. (b)-(c) Transmission spectra of the Fano resonance around $1552.5 \mathrm{~nm}$ versus field transmission coefficients $t_{1}$ and $t_{2}$, respectively. (d)-(e) SR and IL as functions of $t_{1}$ and $t_{2}$, respectively.

Figure 3(a) shows the calculated power transmission spectrum of the zig-zag CSLR resonator. The structural parameters $\left(L_{S L R}=L_{1,2,3,4}=113.75 \mu \mathrm{m}, t_{1}=0.74, t_{2}=0.994\right)$ are designed to achieve a free spectral range (FSR) of about $100 \mathrm{GHz}$ for adjacent resonance peaks (FSR1 $=100.01 \mathrm{GHz}$ and FSR2 $=102.78 \mathrm{GHz}$ ). The transmission spectrum also has periodic Fano resonances. Figures 3(b) and (c) show the transmission spectra for different $t_{1}$ and $t_{2}$, respectively. The calculated SR and IL as functions of $t_{1}$ and $t_{2}$ are depicted in Figs. 3(d) and (e), respectively. Table I compares the ERs, Q factors, ILs, and SRs of the two CSLR resonators in Figs. 2(a) and 3(a). As compared with the parallel CSLR resonator, the zig-zag CSLR resonator shows a better SR, indicating stronger coherent optical mode interferences in the resonant cavities with more SLRs.

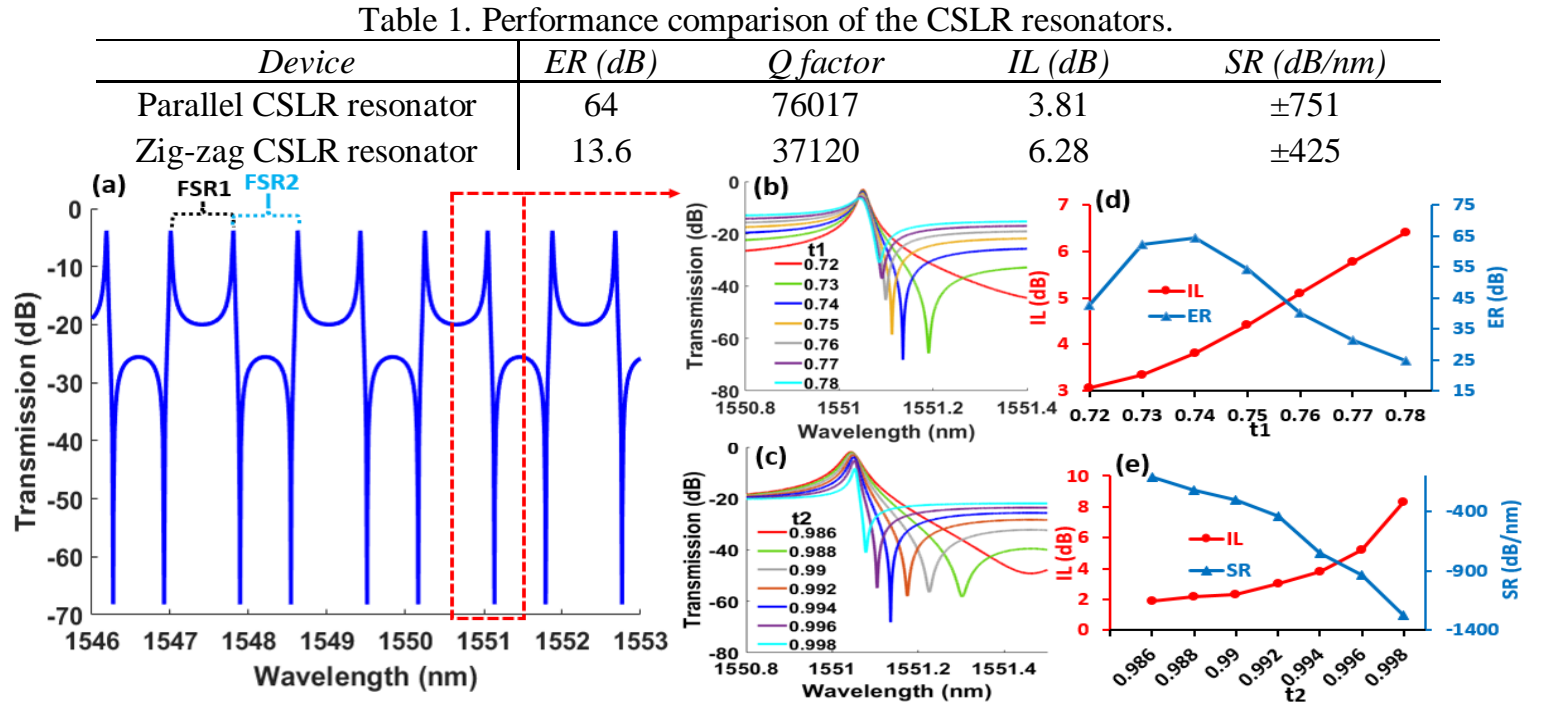

Fig. 3. (a) Calculated transmission spectrum of the zig-zag CSLR resonator. (b)-(c) Transmission spectra of the Fano resonance around 1551 $\mathrm{nm}$ versus field transmission coefficients $t_{1}$ and $t_{2}$, respectively. (d)-(e) SR and IL as functions of $t_{1}$ and $t_{2}$, respectively.

\section{Conclusions}

We investigate Fano resonances in integrated CSLR resonators. By tailoring mode splitting in the CSLR resonators, Fano-like resonances with ultrahigh ERs and SRs are achieved.

\section{References}

[1] M. F. Limonov., et al., "Fano resonances in photonics," Nat. Photon., 11(9), 543, 2017.

[2] E. Kamenetskii., et al., "Fano resonances in optics and microwaves: physics and applications," Springer International Publishing, 2018.

[3] A. E. Miroshnichenko., et al., "Fano resonances in nanoscale structures," Rev. Mod. Phys., 82(3), $2257,2010$.

[4] U. Fano, "Sullo spettro di assorbimento dei gas nobili presso il limite dello spettro d'arco," Il Nuovo Cimento., 12(3), $154,1935$.

[5] U. Fano, "Effects of configuration interaction on intensities and phase shifts," Phys. Rev., 124(6), $1866,1961$.

[6] Z. Wang., et al., "Nano-Fano resonances and topological optics," in Singular and Chiral Nanoplasmonics, 285,2014 ed.

[7] W. Lu, et al., "Switching plasmonic Fano resonance in gold nanosphere-nanoplate heterodimers," Nanoscale, 11(19), 9641, 2019.

[8] K. Lee., et al., "Ultrasensitive biosensors using enhanced Fano resonances in capped gold nanoslit arrays," Sci Rep, 5(1), 8547, 2015.

[9] A. N. Poddubny., et al., "Fano interference governs wave transport in disordered systems," Nature Commun, 3(1), 914, 2012.

[10] C. Grillet, et al., "Characterization and modeling of Fano resonances in chalcogenide photonic crystal membranes", Optics Express 14 (1), 369-376. 2006

[11] D. Freeman, et al., "Chalcogenide Glass Photonic Crystal Devices", Photonic and Electromagnetic Crystal Structures, Photonics and Nanostructures-Fundamentals and Applications, Vol. 6, No. 1, pp 3-11. 2008. doi:10.1016/j.photonics.

[12] J. Wu, et al., "Advanced photonic filters based on cascaded Sagnac loop reflector resonators in SOI nanowires," APL Photonics, 3(4), $046102,2018$.

[13] J. Wu, et al., "Micro-ring resonator quality factor enhancement via an integrated Fabry-Perot cavity," APL Photonics, 2(5), 056103, 2017. 